\title{
Development of an electrochemical impedance analysis program based on the expanded measurement model
}

\author{
Kiyoshi KOBAYASHI, ${ }^{\dagger}$ Yoshio SAKKA and Tohru S. SUZUKI
}

Research Center for Functional Materials, National Institute for Materials Science, 1-2-1 Sengen, Tsukuba, Ibaraki 305-0047, Japan

\begin{abstract}
A new program for electrochemical impedance analysis has been developed based on the expanded measurement model. The main feature of this program is that it allows the user to easily estimate the equivalent circuit, as well as an initial guess of the fitting parameters, through a graphic user interface (GUI). The function to illustrate the partial impedance spectra is

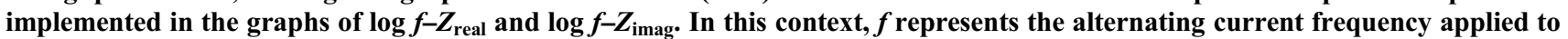
the sample; $Z_{\text {real }}$ and $Z_{\text {imag }}$ represent the real and imaginary components of the impedance, respectively. By employing the GUI supporting function, the impedance spectrum can be analyzed without the use of a pseudo-element such as a constant phase element and with an empirical parameter in the Warburg and Gerischer impedance. Based on the results, it is possible to calculate a discrete distribution of the relaxation times from the optimized parameters.
\end{abstract}

(C2016 The Ceramic Society of Japan. All rights reserved.

Key-words : Electrochemical impedance, Software, Graphic user interface, Discrete distribution of relaxation times

\section{Introduction}

Electrochemical impedance analysis is a powerful technique for separating different processes related to ionic conduction in electrolyte and electrode reactions. ${ }^{1)-6)}$ Nowadays the full spectrum can be analyzed using computer programs developed around $1970.2-4), 7)-10$ ) The key ingredient of such programs is the nonlinear least-squares algorithm for complex functions - namely, the complex nonlinear least-squares (CNLS) method. ${ }^{2), 4), 7), 8)}$ When such programs are combined with the drastic improvements of the past 40 years in the calculation performance of personal computers, the measured impedance spectrum can be analyzed using a program such as LEVM $^{1)-4)}$ with an appropriate equivalent circuit.

Concerning the nonlinear least-squares algorithm, the convergence of fitting strongly depends on the initial guess of the fitting parameters. Hence, it is necessary to set the initial guess of parameters close to their true values. ${ }^{11)-14)}$ Regardless of this, however, the effective support function for guessing the equivalent circuit and the associated parameter values is not implemented in the programs that are widely used today. ${ }^{15)-21)}$

Agarwal et al. reported on electrochemical impedance analysis using the equivalent circuit shown in Fig. 1, which they referred to as the "measurement model." ${ }^{22)}$ The basic concept is that entire impedance spectrum can be thought of as the sum of the partial impedance spectra, each of which reflects a different electrochemical process. According to this analysis, the number of different electrochemical processes is resolved from the impedance dataset as an inverse problem. If this concept is generally expanded to electrochemical systems, it becomes possible to remove difficulty and complexity from the impedance analysis, and the expanded measurement model becomes a guide for constructing a new impedance analysis program. Furthermore, the fitting results based on the measurement model become a guide

\footnotetext{
Corresponding author: K. Kobayashi; E-mail: KOBAYASHI. kiyoshi@nims.go.jp
}

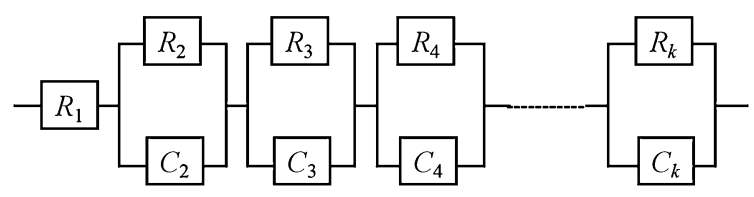

Fig. 1. Equivalent circuit model of the measurement model.

for the expansion to another process model that is constructed to help understand electrochemical processes and to explain the measured spectrum. However, a program for analyzing impedance based on the expanded measurement model has not been developed yet.

In this study, we develop a new program for analyzing electrochemical impedance based on the expanded measurement model. The primary motivation of this program is to offer an easy estimation of the equivalent circuit model and the initial values of the parameters. This is achieved by facilitating an interaction between the plotted graphs and the user through the graphic user interface (GUI), ultimately removing certain difficulties from the analysis. In addition, we demonstrate a different impedance analysis without the use of a pseudo-element such as a constant phase element (CPE) even when the measured spectrum is different from the ideal spectrum. By employing this method, the discrete distribution of relaxation times can be analyzed from the fitting parameters.

\section{Program design}

\subsection{Equivalent circuit model}

An inherent ambiguity surrounds the equivalent circuit in that it corresponds to many distinct impedance spectra. ${ }^{1), 6), 23)}$ Among the equivalent models, the measurement model ${ }^{22}$ is selected in this study because it is constructed so as to resolve the electrochemical processes in the measured impedance spectrum. Moreover, various spectral shapes can be fitted by the measurement model, and can be expanded to an electrochemical system when 


\section{$Z_{1} Z_{2}-Z_{3}-Z_{k}$}

Fig. 2. Equivalent circuit model for the expanded measurement model. The $Z_{k}$ values represent the partial impedance elements, which are listed in Table 1.

Table 1. List of the partial impedance elements of the expanded measurement model. The index represents the label of the partial impedance element. $f$ and $j$ represent the frequency and imaginary unit, respectively. $Z_{k}$ represents the partial impedance. The ideal $\alpha$ values for $W$-trd, $W$-md, $W$-nmd, $G-I$, and $G-I I$ are provided in parentheses

\begin{tabular}{|c|c|c|c|}
\hline Element Name & Index & $\begin{array}{c}\text { Fitting } \\
\text { Parameter(s) }\end{array}$ & $Z_{k}=$ \\
\hline Resistor & $R$ & $\mathrm{R}$ & $\mathrm{R}$ \\
\hline Inductor & $L$ & $\mathrm{~L}$ & $\mathrm{~L} 2 \pi f j$ \\
\hline Capacitor & $C$ & $\mathrm{C}$ & $\frac{1}{\mathrm{C} 2 \pi f j}$ \\
\hline Constant Phase Element & $C P E$ & $\mathrm{~T}, \alpha$ & $\frac{1}{\mathrm{~T}(2 \pi f j)^{\alpha}}$ \\
\hline Debye-type & $R / / C$ & $\mathrm{R}, \mathrm{T}$ & $\frac{\mathrm{R}}{1+\mathrm{RC} 2 \pi f j}$ \\
\hline Inductive Debye-type & $R / / L$ & $\mathrm{R}, \mathrm{L}$ & $\frac{\mathrm{RL} 2 \pi f j}{\mathrm{R}+\mathrm{L} 2 \pi f j}$ \\
\hline Cole-Cole-type & $R / / C P E$ & $\mathrm{R}, \mathrm{T}, \alpha$ & $\frac{\mathrm{R}}{1+\mathrm{RT}(2 \pi f j)^{\alpha}}$ \\
\hline Traditional Warburg & $W$-trd & $\mathrm{T}, \alpha(=0.5)$ & $\frac{1}{\mathrm{~T}(2 \pi f j)^{\alpha}}$ \\
\hline $\begin{array}{l}\text { Mass dissipative } \\
\text { Warburg }\end{array}$ & $W-m d$ & $\mathrm{R}, \mathrm{T}, \alpha(=0.5)$ & $\mathrm{R} \frac{\tanh (\mathrm{T} 2 \pi f j)^{\alpha}}{(\mathrm{T} 2 \pi f j)^{\alpha}}$ \\
\hline $\begin{array}{l}\text { Non mass dissipative } \\
\text { Warburg }\end{array}$ & $W$-nmd & $\mathrm{R}, \mathrm{T}, \alpha(=0.5)$ & $\mathrm{R} \frac{\operatorname{coth}(\mathrm{T} 2 \pi f j)^{\alpha}}{(\mathrm{T} 2 \pi f j)^{\alpha}}$ \\
\hline Gerischer type-I & $G-I$ & $\mathrm{R}, \mathrm{T}, \alpha(=1)$ & $\frac{\mathrm{R}}{\sqrt{1+(\mathrm{T} 2 \pi f i)^{\alpha}}}$ \\
\hline Gerischer type-II & $G-I I$ & $\mathrm{R}, \mathrm{T}, \alpha(=0.5)$ & $\frac{\mathrm{R}}{(1+\mathrm{T} 2 \pi f j)^{\alpha}}$ \\
\hline
\end{tabular}

one takes into account special impedances that arise in electrochemical systems, such as the Warburg impedance and the Gerischer impedance. ${ }^{24)}$ In particular, the Warburg impedance and the Gerischer impedance are theoretically derived impedances based on Fick's second law, as well as the application of this law to chemical reaction kinetics. ${ }^{24)}$ These impedances are usually applied to explain the electrode's impedance.

The equivalent circuit of the expanded measurement model is presented in Fig. 2 using the partial impedance element $\left(Z_{k}\right)$. In this model, $Z_{k}$ is distinguished by the type of relaxation, and it represents in three-dimensional impedance space (i.e., $\log f-Z_{\text {real }}-$ $Z_{\text {imag }}$ space), where $f$ represents the alternating current frequency applied to the sample; $Z_{\text {real }}$ and $Z_{\text {imag }}$ represent the real and imaginary components of the impedance, respectively. In order to account for an electrochemical process, at least twelve elements must be considered if two types of generalized Gerischer impedance are accepted. ${ }^{18)}$ The partial impedance elements and their theoretical equations are listed in Table 1. ${ }^{8), 12), 18)}$ Because of the serial connection of the $Z_{k} \mathrm{~s}$, the partial spectra of each $Z_{k}$, as well as the total impedance spectrum, can be calculated in the case of the expanded measurement model. ${ }^{22}$ ) This feature indicates that the contribution of each $Z_{k}$ to the total impedance spectrum can be visualized in the $\log f-Z_{\text {real }}$ and $\log f-Z_{\text {imag }}$ plots. ${ }^{22)}$ In developing the analysis program, we coded a function that graphs the total impedance spectrum and the partial impedance spectra automatically.

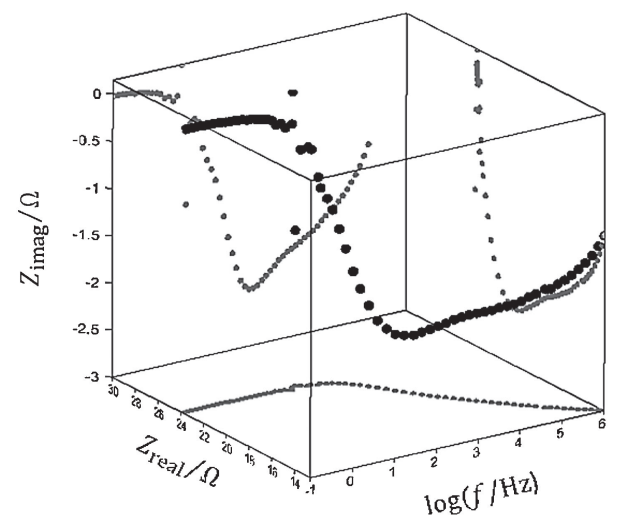

Fig. 3. Three-dimensional representation of impedance (closed circles) The projected curves on the $\log f-Z_{\text {real }}, \log f-Z_{\text {imag }}$, and $Z_{\text {real }}-Z_{\text {imag }}$ surfaces are plotted by gray circles.

\subsection{Displaying graphs for analysis support}

The impedance spectrum is a three-dimensional curve in $\log f-$ $Z_{\text {real }}-Z_{\text {imag }}$ space, ${ }^{1), 4), 25)}$ and the plots of $\log f-Z_{\text {real }}, \log f-Z_{\text {imag }}$, and $Z_{\text {real }}-Z_{\text {imag }}$ correspond to the projected curves on each twodimensional plane, as shown in Fig. 3. Because the KramersKronig relations hold for the impedance spectrum of the expanded measurement model, ${ }^{5), 22)}$ one degree of freedom is lost in the three-dimensional relationship. Therefore, at least two curves from the $\log f-Z_{\text {real }}, \log f-Z_{\text {imag }}$, and $Z_{\text {real }}-Z_{\text {imag }}$ plots are necessary to obtain complete information regarding the spectrum. Therefore, for impedance analysis that proceeds by checking each graph, the three plots of $\log f-Z_{\text {real }}, \log f-Z_{\text {imag }}$, and $Z_{\text {real }}{ }^{-}$ $Z_{\text {imag }}$ should preferably displayed in one main window.

One empirical parameter related to the distribution of relaxation times in the Cole-Cole-type element can be ideally estimated from the slope of the $\log f-\log \left|Z_{\text {imag }}\right|$ plot, ${ }^{1), 26)}$ where

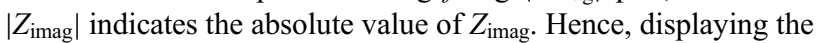
plot of $\log f-\log \left|Z_{\text {imag }}\right|$, as well as the plot of its slope $(\log f$ $\left.\partial \log f / \partial \log \left|Z_{\text {imag }}\right|\right)$, is helpful in carrying out impedance analysis.

Moreover, in order to develop a new program for electrochemical impedance analysis, a graphical display of the quantities related to the goodness of fit of the model is helpful for judging whether or not the model can sufficiently explain the measured spectrum. In this program, the residuals are plotted in addition to the $\log f-Z_{\text {real }}, \log f-Z_{\text {imag }}$, and $Z_{\text {real }}-Z_{\text {imag }}$ graphs. The residual impedance $(\Delta Z)$ at each frequency is calculated by taking the difference between the measured impedance $\left(Z_{\text {meas }}\right)$ and the impedance calculated using the employed equivalent circuit $\left(Z_{\text {model }}\right)$ as follows:

$$
\Delta Z=Z_{\text {meas }}-Z_{\text {model }} .
$$

Because $\Delta Z$ is a complex number, it is necessary to divide the real and imaginary components of $\Delta Z$ for graphical display, which are represented by $\Delta Z_{\text {real }}$ and $\Delta Z_{\text {imag }}$, respectively. The main window, shown in Fig. 4, is designed to take all of these graphical requirements into account.

\subsection{Estimation method for the equivalent circuit and the initial guess of the parameters}

Although modern scripting and general-purpose programing languages support the nonlinear least-squares function (e.g., the Levenberg-Marquardt algorithm and so on) as a default module or extended module that can be applied by installing extension libraries, this is applicable to real-variable functions only. Therefore, it is necessary to confirm that these nonlinear least-squares 


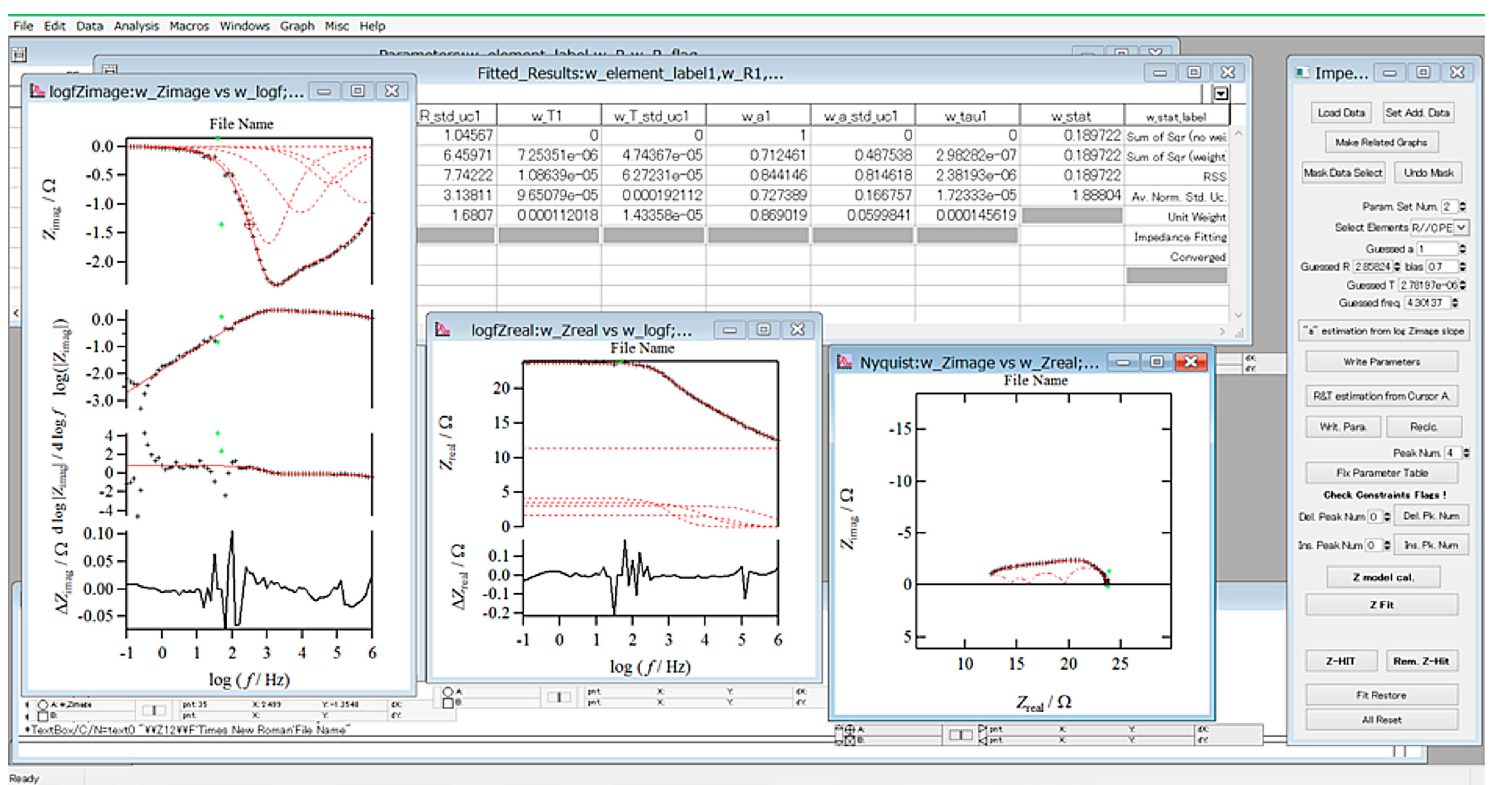

Fig. 4. Overview of the developed software. The word "Slope" indicates the numerically calculated slope of the $\log \left(\left|Z_{\text {imag }}\right|\right)-$ $\log f$ curve: namely, $\partial \log \left(\left|Z_{\text {imag }}\right|\right) / \partial \log f$.

modules can be applied as the CNLS method on scripting and general-purpose programing languages. To this end, we first coded minimal programs for impedance analysis on the programing languages IgorPro, ${ }^{27)}$ Scilab, ${ }^{28)}$ Python, ${ }^{29)} \mathrm{R},{ }^{30)}$ and GNU Octave. ${ }^{31)}$ All of these programing languages and their modules were found to be sufficient for impedance fitting by adequate coding of the user-defined function related to the residual. In the case of no data weighting, the residual function is given by:

$$
f(\cdots)=\sqrt{\left(\Delta Z_{\text {real }}\right)^{2}+\left(\Delta Z_{\text {imag }}\right)^{2}} .
$$

With respect to the arguments of the user-defined function $(f)$, some restrictions exist in order to apply the nonlinear leastsquares modules. Therefore, the coding manner strongly depends on the language used.

Furthermore, it is necessary to provide an appropriate model (i.e., an equivalent circuit) and input the values as proper initial guesses of the fitting parameters in order to converge the calculations. In the case of the expanded measurement model, initial guess estimations for the fitting parameters are relatively easy to obtain by considering the features of the partial impedance elements, because the total impedance spectrum is treated as the sum of the partial impedance spectra.

Theoretical equations of the partial impedance elements are presented in Table 1. By combining the theoretical relationships of each partial impedance element and the geometrical features of the $Z_{\text {real }}-Z_{\text {imag }}$ plot, ${ }^{1), 4)}$ the estimation methods for the initial guess of the fitting parameters can be distinguished into two types: ${ }^{18)}$ (I) parameters are estimated from a typical data point that is governed by the partial impedance element in question; and (II) parameters are estimated from an impedance data point that is close to the characteristic impedance that is characterized by the partial impedance element.

The partial impedance elements whose projections are either a single point or a one-dimensional line in the $Z_{\text {real }}-Z_{\text {imag }}$ plane belong to type (I). In the case of type-(I) elements, the fitting parameters can be estimated from a typical data point for $R, L$, or $C$; on the other hand, for the $C P E$ and traditional Warburg ( $W$-trd $)$ elements, a typical data point must be combined with the slope of the line in the $Z_{\text {real }}-Z_{\text {imag }}$ plot.

For type-(II) elements, the partial impedance spectrum is characterized by the characteristic impedance, which refers to the impedance at the characteristic frequency. The characteristic frequency is the frequency that is associated with the fitting parameters and a factor of $2 \pi$. In other words, if we employ the angular frequency $(\omega)$ instead of the frequency $(f)$, the $2 \pi$ term disappears according to the relation $\omega=2 \pi f$. The type-(II) elements are the Debye-type $(R / / C)$ element, the inductive Debye-type $(R / / L)$ element, the Cole-Cole-type $(R / / C P E)$ element, the mass-dissipative, finite-diffusion-length Warburg impedance $(W-m d)$ element, ${ }^{18), 24), 32)}$ the no-mass-dissipation, finitediffusion-length Warburg impedance $(W-n m d)$ element, ${ }^{18), 24), 32)}$ as well as two varieties of the generalized Gerischer impedance ( $G-I$ and $G-I I)$ element. ${ }^{18), 24), 32)}$ By combining the equations for the partial impedance elements with their geometric relationships in the $Z_{\text {real }}-Z_{\text {imag }}$ plane, ${ }^{1), 4)}$ the guessed parameters can be calculated from the characteristic impedance using the equations in Table 2. (1),4),6),18) The characteristic impedance point is easily distinguishable on the $\log f-Z_{\text {imag }}$ and $Z_{\text {real }}-Z_{\text {imag }}$ plots.

Regarding the $R / / C P E, W-m d, W-n m d, G-I$, and $G-I I$ elements, an empirical parameter, represented by $\alpha$ (Table 1 ), as well as the characteristic impedance point are necessary for the initial guess. Although the $\alpha$ value can ideally be estimated from the constant slope of the $\log f-\log \left|Z_{\text {imag }}\right|$ plot, ${ }^{1), 26)}$ this method is usually difficult owing to noise contained in the data, as well as the convolution of the several partial impedance spectra. In such cases, a simple and effective procedure is to calculate the partial impedance using the impedance datum close to the characteristic impedance with the appropriate $\alpha$ value; next, the partial impedance can be recalculated after changing the $\alpha$ value in order to bring it closer to the measured data. By comparing the measured impedance data against the calculated data using the guessed parameters, the appropriate initial guess values can be easily determined.

For both types of elements, the initial guess can be made by selecting the element's name from a pop-up menu, selecting the typical impedance point for the type-(I) element and the point 
Table 2. List of the equations for calculating the initial guess of the fitting parameters for the type-(II) partial impedance elements. Theoretical relationships between the characteristic frequency $\left(f_{\mathrm{c}}\right)$ and fitting parameters $(R, C, L, T$, and $\alpha)$ are listed in the $f_{\mathrm{c}}$ column. The $\alpha_{\mathrm{g}}, \mathrm{R}_{\mathrm{g}}$, and $\mathrm{C}_{\mathrm{g}}, \mathrm{L}_{\mathrm{g}}$, or $\mathrm{T}_{\mathrm{g}}$ columns indicate the relationships to the guessed $\alpha, R, C, L$, and $T$ values using $f_{\mathrm{c}}, \alpha_{\mathrm{g}}$, the absolute value of $Z_{\text {imag }}$ at the $f_{\mathrm{c}}\left(\left|Z_{\text {imag_c }}\right|\right)$, and the frequency $(f)$

\begin{tabular}{|c|c|c|c|c|}
\hline Index & $f_{\mathrm{c}}$ & $\alpha_{\mathrm{g}}$ & $\mathrm{R}_{\mathrm{g}}$ & $\mathrm{L}_{\mathrm{g}}, \mathrm{C}_{\mathrm{g}}$, or $\mathrm{T}_{\mathrm{g}}$ \\
\hline \multirow{2}{*}{$R / / C$} & 1 & \multirow{2}{*}{ Not Necessary } & \multirow{2}{*}{$2\left|Z_{\text {imag_c }}\right|$} & 1 \\
\hline & $\overline{2 \pi \mathrm{RC}}$ & & & $\overline{2 \pi \mathrm{R}_{\mathrm{g}} f_{\mathrm{c}}}$ \\
\hline \multirow{2}{*}{$R / / L$} & $\mathrm{R}$ & \multirow{2}{*}{ Not Necessary } & \multirow{2}{*}{$2\left|Z_{\text {imag_c }}\right|$} & $\mathrm{R}_{\mathrm{g}}$ \\
\hline & $\overline{2 \pi \mathrm{L}}$ & & & $\overline{2 \pi f_{\mathrm{c}}}$ \\
\hline \multirow{2}{*}{$R / / C P E$} & 1 & $d \log \left|Z_{\text {imag }}\right|$ & $2\left|Z_{\text {imag_c }_{-c}}\right| \cos \left\{\pi\left(1-\alpha_{\mathrm{g}}\right) / 2\right\}$ & 1 \\
\hline & $\overline{2 \pi(\mathrm{RT})^{1 / \alpha}}$ & $d \log f$ & $1-\sin \left\{\pi\left(1-\alpha_{\mathrm{g}}\right) / 2\right\}$ & $\overline{\mathrm{R}_{\mathrm{g}}\left(2 \pi f_{\mathrm{c}}\right)^{\alpha_{\mathrm{g}}}}$ \\
\hline \multirow{2}{*}{$W-m d$} & 1 & $d \log \left|Z_{\text {imag }}\right|$ & \multirow{2}{*}{$2.54\left|Z_{\text {imag_c }}\right|$ at $\alpha_{\mathrm{g}}=0.5^{\dagger}$} & $2.54 \dagger$ \\
\hline & $\overline{2 \pi \mathrm{T}^{1 / \alpha}}$ & $d \log f$ & & $\overline{f_{\mathrm{c}}}$ \\
\hline \multirow{2}{*}{$W$-nmd } & 1 & $d \log \left|Z_{\text {imag }}\right|$ & \multirow{2}{*}{$3\left|Z_{\text {imag_c }}\right|$ at $\alpha_{\mathrm{g}}=0.5^{\dagger}$} & 1 \\
\hline & $\overline{2 \pi \mathrm{T}^{1 / \alpha}}$ & $d \log f$ & & $\overline{f_{\mathrm{c}}}$ \\
\hline \multirow{2}{*}{$G-I$} & 1 & $d \log \left|Z_{\text {imag }}\right|$ & \multirow{2}{*}{$2.6\left|Z_{\text {imag_c }}\right|$ at $\alpha_{\mathrm{g}}=1$} & 2.6 \\
\hline & $\overline{2 \pi \mathrm{T}^{1 / \alpha}}$ & $d \log f$ & & $\overline{f_{\mathrm{c}}}$ \\
\hline \multirow{2}{*}{$G-I I$} & 1 & $d \log \left|Z_{\text {imag }}\right|$ & \multirow{2}{*}{$2.6\left|Z_{\text {imag_c }}\right|$ at $\alpha_{\mathrm{g}}=0.5$} & $2.6+$ \\
\hline & $\overline{2 \pi \mathrm{T}^{1 / \alpha}}$ & $d \log f$ & & $\overline{f_{\mathrm{c}}}$ \\
\hline
\end{tabular}

${ }^{\dagger}$ These are the empirical equations originally given in Refs. 9, 18. However, the guessed values are not adequate when using the original relationships. Hence, the coefficient values are modified based on several numerical calculations.

close to the characteristic impedance point for the type-(II) element on the $\log f-Z_{\text {imag }}$ or $\log f-Z_{\text {real }}$ graphs, and setting an appropriate $\alpha$ value. These tasks can be performed on the button control panel shown on the right side of Fig. 4, and with the cursor on the graphs. The impedance spectrum calculated using the initial guess parameters can be drawn in the graphs using several buttons. Moreover, it is possible to change the initial guess values, recalculate the impedance with the new parameters, and redraw that result. Hence, users can easily create an initial model by checking the graphs.

\subsection{Selection of the programming language and the nonlinear least-squares module}

For the actual programming, it is necessary for the programmable commands to be related to various GUIs, as well as various kinds of numerical calculation - such as double precision complex and real numbers, nonlinear least-squares analysis, and so on. Hence, an integrated language that can handle not only the specified scientific calculation but also the graphic operation is preferable in this study. Based on the background explained above, we selected the IgorPro software. ${ }^{27)}$

With respect to the nonlinear least-squares module, we used the Levenberg-Marquardt algorithm within the orthogonal distance regression library pack (ODR95), ${ }^{33), 34)}$ which is built into IgorPro.

With respect to the error of the fitting parameters, the fractional standard uncertainty is calculated from the fractional covariance matrix when the error values of the $Z_{\text {real }}$ and $Z_{\text {imag }}$ dataset at each frequency are not known. ${ }^{35)-37)}$ Hence, the standard uncertainty of the fitting parameters is calculated by the diagonal terms of the fractional covariance matrix, the residual sum of squares, the number of data points, and the number of parameters. ${ }^{35)}$

\section{Experimental procedures}

One impedance spectrum was measured and analyzed by our program using a solid oxide fuel (SOFC)-type cell. The SOFCtype cell was fabricated using yttria-stabilized zirconia (TZ-8Y, Tosoh Corp., Japan), lanthanum cobaltite $\left(\mathrm{LaCoO}_{3}\right)$ synthesized by a citrate combustion method, ${ }^{38)}$ and platinum paste (TR-7907, Tanaka Kikinzoku Kougyo, Japan). The three-terminal config- uration cell was fabricated by the same procedure used in a previous study. ${ }^{38)}$

The impedance spectrum of the SOFC-type cell was measured at $1073 \mathrm{~K}$ under air using the potentio/galvanostat built-in frequency response analyzer (Reference-600, Gamry Instruments, USA). The frequency ranged from $1 \mathrm{MHz}$ to $0.1 \mathrm{~Hz}$ with 10 points per order of magnitude arranged on a logarithmic scale. The amplitude voltage was $50 \mathrm{mV}$. The spectrum was collected in a high frequency to low frequency manner.

\section{Analysis results}

The impedance spectrum collected by three terminal cells using yttria-stabilized zirconia, a lanthanum cobaltite cathode, a platinum anode, and a reference electrode is shown in Fig. 5. This spectrum is usually fitted using the $R / / C P E$ element because of its non-ideal spectral shape. The most adequate model, which uses the $R$ and $R / / C P E$ elements, is shown in Fig. 6. Although the fitting spectrum (Fig. 5) seems to turn out well, the fitting parameters are found to be insufficient because the standard uncertainty values of R2, T2, R3, and T3 are larger than the corresponding parameters listed in Table 3. Moreover, we confirmed that the standard uncertainty values did not improve even when more $R / / C P E$ elements were added in the circuit. The physical meaning of the $\mathrm{T}$ parameter in $C P E$ is ambiguous because the $\alpha$ parameter value is included in its dimensions $\left(\mathrm{F} \cdot \mathrm{s}^{1-\alpha}\right)$. Based on the dimensions of $\mathrm{T}$, it is difficult to compare the $\mathrm{T}$ values optimized from different impedance spectra when the corresponding $\alpha$ value is different.

Meanwhile, the spectrum can be fitted without using a $C P E$ (Fig. 7) by employing the equivalent circuit shown in Fig. 8. Although the least-squares procedure diverged when the initial parameter values were significantly different from the true values, adequate values could be easily selected by employing the GUI supporting function of our software. In addition, all of the standard uncertainty values are smaller than the corresponding parameters shown in Table 4. Regarding to $R / / C$ elements, the relaxation time of each $R / / C$ element is calculated from the optimized parameters. Hence, the discrete distribution of relaxation times is analyzed as function of the corresponding $\mathrm{R}$ values, as shown in Fig. 9. In this software, displaying function of the 
Table 3. Fitting parameters of the impedance spectrum shown in Fig. 5 using the equivalent circuit in Fig. 6. Standard uncertainty values are provided in parentheses. $\alpha 1, \alpha 2$, and $\alpha 3$ are dimensionless parameters

\begin{tabular}{|c|c|c|c|c|c|c|}
\hline$R 1 / \Omega$ & $R 2 / \Omega$ & $T 2 / \mathrm{F} \cdot \mathrm{s}^{1-\alpha 2}$ & $\alpha 2$ & $R 3 / \Omega$ & $T 3 / \mathrm{F} \cdot \mathrm{s}^{1-\alpha 3}$ & $\alpha 3$ \\
\hline $\begin{array}{l}11.0 \\
(0.6)\end{array}$ & $\begin{array}{l}5.3 \\
(6)\end{array}$ & $\begin{array}{c}7 \times 10^{-5} \\
\left(2 \times 10^{-4}\right)\end{array}$ & $\begin{array}{c}0.6 \\
(0.2)\end{array}$ & $\begin{array}{c}3 \\
(7)\end{array}$ & $\begin{array}{c}1 \times 10^{-4} \\
\left(3 \times 10^{-4}\right)\end{array}$ & $\begin{array}{c}0.7 \\
(0.4)\end{array}$ \\
\hline$R 4 / \Omega$ & $\mathrm{T} 4 / \mathrm{F} \cdot \mathrm{s}^{1-\alpha 4}$ & $\alpha 4$ & & & & \\
\hline $\begin{array}{c}4 \\
(2)\end{array}$ & $\begin{array}{r}1.1 \times 10^{-4} \\
\left(1 \times 10^{-5}\right)\end{array}$ & $\begin{array}{c}0.87 \\
(0.07)\end{array}$ & & & & \\
\hline
\end{tabular}

(b)

(a)
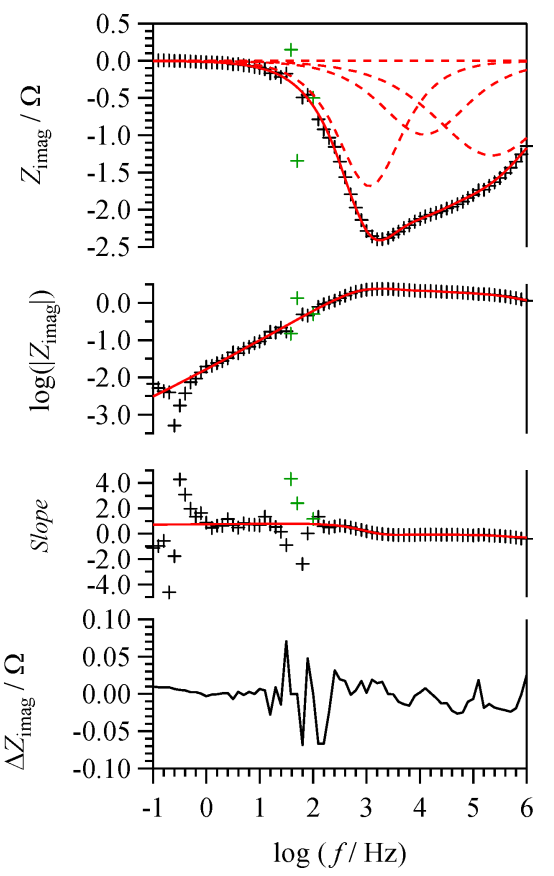

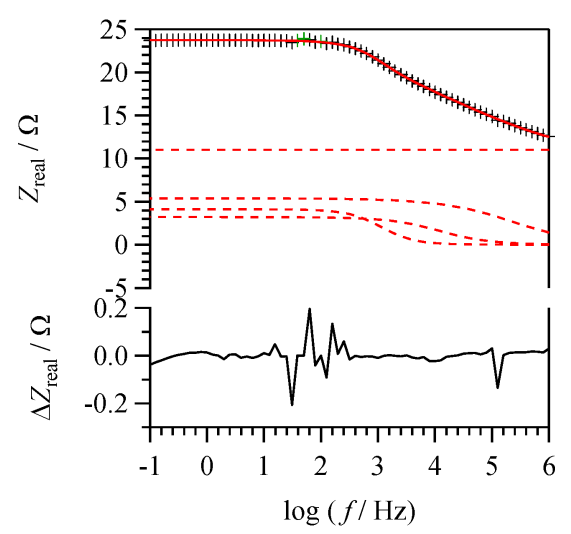

(c)

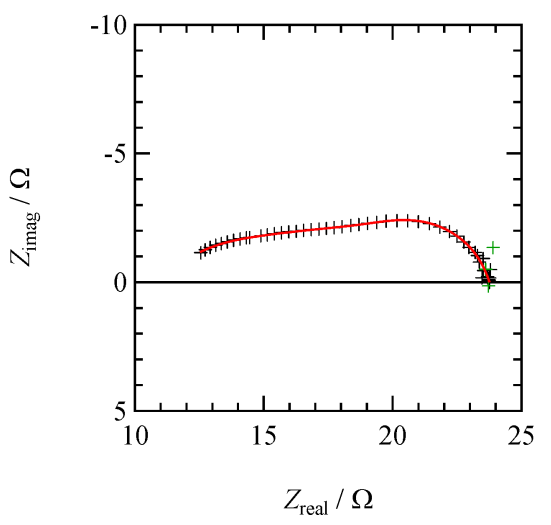

Fig. 5. Impedance spectrum of the three terminal cells constructed of yttria-stabilized zirconia, a lanthanum cobaltite cathode, a platinum anode, and a platinum reference electrode. In sub-window (a), four graphs are plotted as function of $\log f$. The terms of $Z_{\text {imag }}$ and $\left|Z_{\text {imag }}\right|$ represent the imaginary part of the impedance and its absolute value. The slope and $\Delta Z_{\text {imag }}$ represent the slope of the $\log \left|Z_{\text {imag }}\right|-\log f$ curve and the difference between the imaginary parts of the measured and calculated impedance. In sub-window (b), two graphs are plotted as a function of $\log f$. The vertical terms of $Z_{\text {real }}$ and $\Delta Z_{\text {real }}$ represent the real part of the impedance and the difference between the real parts of the measured and calculated impedance, respectively. In sub-window (c), the relationship between $Z_{\text {real }}$ and $Z_{\text {imag }}$ is plotted. The fitting results obtained by using the equivalent circuit shown in Fig. 6 are plotted using red curves. The total fitting curves are plotted using red solid lines. The partial impedance is plotted using red broken curves. The green markers indicate the masked data, which are omitted from the least-squares fitting because of the spike noise.

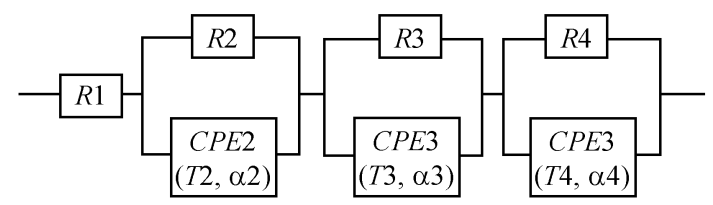

Fig. 6. Equivalent circuit model used for the spectrum in Fig. 5. $R_{\mathrm{n}}, T_{\mathrm{n}}$, and $\alpha_{\mathrm{n}}(\mathrm{n}=1$ to 4$)$ are the fitting parameters. discrete distribution of the relaxation time is also implemented.

\section{Conclusion}

New software for impedance analysis was developed. The basic design concept of this program is that it allows for the user to easily understand the impedance spectrum. The unique functions and features are as follows. (I) Users can see and check the spectral information from the curve shapes using several plots shown in one window. (II) The measured spectrum can be 
Table 4. Fitting parameters of the impedance spectrum shown in Fig. 7 using the equivalent circuit in Fig. 8. Standard uncertainty values are provided in parentheses

\begin{tabular}{|c|c|c|c|c|c|c|}
\hline$R 1 / \Omega$ & $R 2 / \Omega$ & $C 2 / \mathrm{F}$ & $R 3 / \Omega$ & $C 3 / \mathrm{F}$ & $R 4 / \Omega$ & $C 4 / \mathrm{F}$ \\
\hline $\begin{array}{l}11.8 \\
(0.3)\end{array}$ & $\begin{array}{c}1.7 \\
(0.3)\end{array}$ & $\begin{array}{r}1.2 \times 10^{-7} \\
\left(4 \times 10^{-8}\right)\end{array}$ & $\begin{array}{c}1.9 \\
(0.4)\end{array}$ & $\begin{array}{c}7 \times 10^{-7} \\
\left(4 \times 10^{-7}\right)\end{array}$ & $\begin{array}{c}2.1 \\
(0.4)\end{array}$ & $\begin{array}{r}3 \times 10^{-6} \\
\left(1 \times 10^{-6}\right)\end{array}$ \\
\hline$R 5 / \Omega$ & $C 5 / \mathrm{F}$ & $R 6 / \Omega$ & C6/F & $R 7 / \Omega$ & $C 7 / \mathrm{F}$ & \\
\hline $\begin{array}{c}2.2 \\
(0.2)\end{array}$ & $\begin{array}{r}1.5 \times 10^{-5} \\
\left(3 \times 10^{-6}\right)\end{array}$ & $\begin{array}{c}3.3 \\
(0.2)\end{array}$ & $\begin{array}{r}4.7 \times 10^{-5} \\
\left(6 \times 10^{-6}\right)\end{array}$ & $\begin{array}{c}0.6 \\
(0.1)\end{array}$ & $\begin{array}{r}1.5 \times 10^{-3} \\
\left(5 \times 10^{-4}\right)\end{array}$ & \\
\hline
\end{tabular}

(b)

(a)
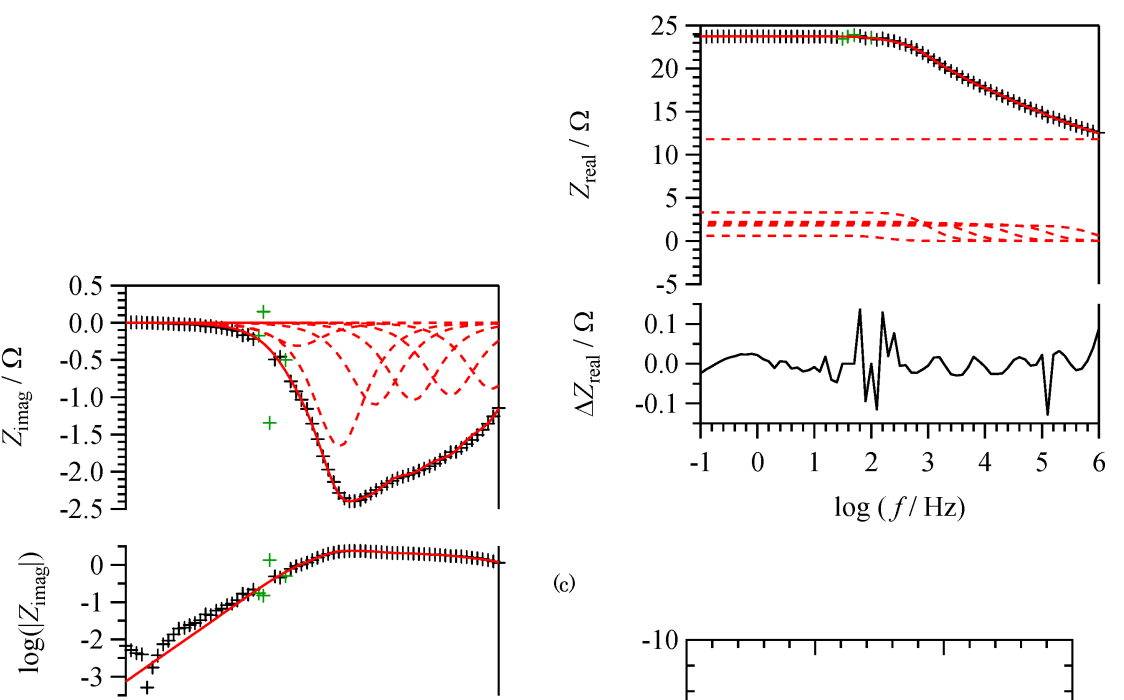

(c)
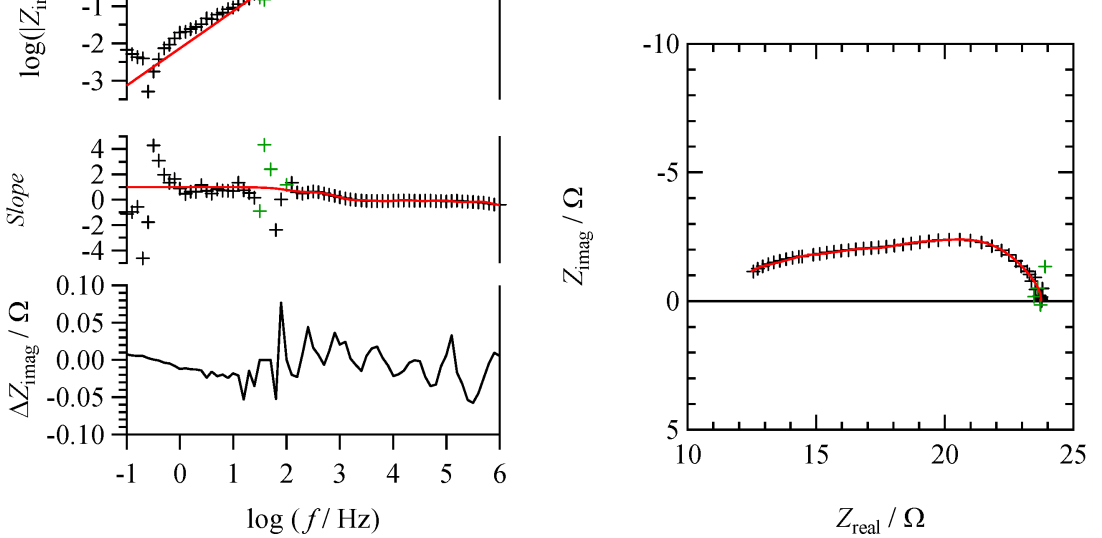

Fig. 7. Fitting results of the spectrum shown in Fig. 5 obtained by employing the equivalent circuit shown in Fig. 8 . The explanations of graphs (a)-(c) are identical to those in Fig. 5. The total fitting curves are plotted using red solid lines. The partial impedance is plotted using red broken curves.

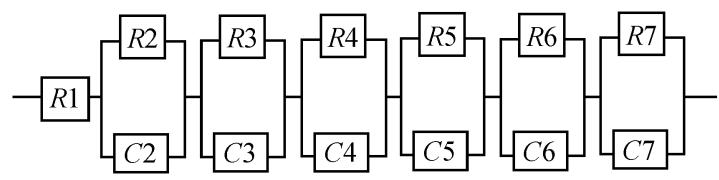

Fig. 8. Equivalent circuit model used for the analysis in Fig. 7. $R_{\mathrm{n}}$ and $C_{\mathrm{n}}(\mathrm{n}=1$ to 7$)$ are the fitting parameters.

expressed as the sum of the partial impedance spectra; this corresponds to the expanded measurement model. (III) A new GUI is proposed for constructing the equivalent circuit model and for evaluating the initial guess of the parameters.

By utilizing the equivalent circuit model and the GUI function in this manner, the impedance spectrum can be fitted without the use of pseudo-elements or empirical parameters, even for the case of a non-ideal spectrum. Accordingly, this method makes it pos-

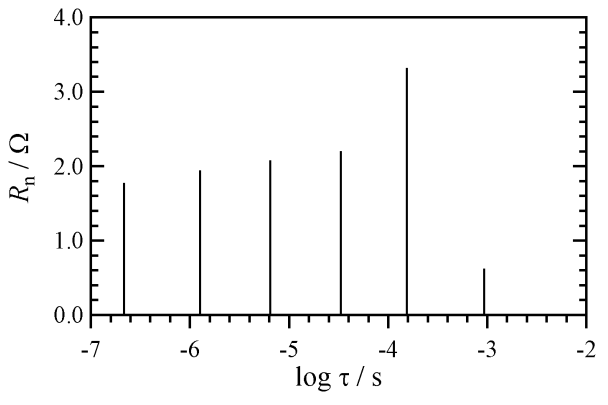

Fig. 9. Discrete distribution of relaxation times calculated by the analysis in Fig. 7. 
sible to analyze the discrete distribution of relaxation times.

Acknowledgement This work was supported by JSPS KAKENHI Grant Number 26420732.

\section{References}

1) E. Barsoukov and J. R. Macdonald, "Impedance Spectroscopy", Second Edition, John Wiley \& Sons Inc., New Jersey (2005).

2) J. R. Macdonald and J. A. Garber, J. Electrochem. Soc., 124, 1022-1030 (1977).

3) J. R. Macdonald, J. Schoonman and A. P. Lehnen, Solid State Ionics, 5, 137-140 (1981).

4) J. R. Macdonald, J. Schoonman and A. P. Lehnen, J. Electroanal. Chem., 131, 77-95 (1982).

5) B. A. Boukamp, Solid State Ionics, 169, 65-73 (2004).

6) D. D. Macdonald, Electrochim. Acta, 51, 1376-1388 (2006).

7) R. J. Sheppard, B. P. Jordan and E. H. Grant, J. Phys. D., 3, 1759-1764 (1970)

8) R. J. Sheppard, J. Phys. D., 6, 790-794 (1973).

9) B. A. Boukamp, Solid State Ionics, 11, 339-346 (1984).

10) S. P. Manoharan, S. Birlasekaran and C. V. Suryanarayana, Bull. Electrochem., 2, 509-513 (1986).

11) A. Lasia, "Electrochemical impedance spectroscopy and its applications", Springer, New York (2014).

12) M. Orazem and E. B. Tribollet, "Electrochemical impedance spectroscopy", John Wiley \& Sons, Inc., New Jersey (2008).

13) V. F. Lvovich, "Impedance spectroscopy", John Wiley \& Sons, Inc., New Jersey (2012).

14) W. H. Press, S. A. Teukolsky, W. T. Vetterling and B. P. Flannery, "Numerical Recipes in C", Cambridge University Press, Cambridge (1992)

15) J. R. Macdonald, LEVMW (free software), http:// jrossmacdonald.com.

16) Scribner Associate Incorp, $\mathrm{ZView}^{\circledR}$ for Windows (Commercial software), http://www.scribner.com/software/generalelectrochemistry/68-general-electrochemistr/376-zview-forwindows.

17) Gamry Instruments, Gamry Echem Analyst (Commercial software), http://www.gamry.com/.

18) BioLogic Science Instruments, EC-Lab ${ }^{\circledR}$ (Commercial software) and EC-Lab ${ }^{\circledR}$ software User's Manual, http://www. bio-logic.info/potentiostat-electrochemistry-ec-lab/.
19) EChem Software, ZSimpWin (Commercial software), http:// www.echemsw.com/.

20) WonATech, ZMAN ${ }^{\mathrm{TM}}$ (Commercial software), http://www. xenosystem.com/zman/.

21) S. Koch, Echemea Analytical (Open source free software), http://www.elchemea.dk/.

22) P. Agarwal, M. E. Orazem and L. H. Garcia-rubio, J. Electrochem. Soc., 139, 1917-1927 (1992).

23) A. Lasia, Electrochemical impedance spectroscopy and its application, in: B. E. Conway, J. O M. Bockris and R. White (Eds.), "Modern Aspects of Electrochemistry", vol. 32, Ch. 2, Kluwer academic press, New York, p. 143 (1992).

24) K. Kobayashi, T. Terabe, T. Sukigara and Y. Sakka, Solid State Ionics, 249, 78-85 (2013).

25) A. S. Bondarenko, Anal. Chim. Acta, 743, 41-50 (2012).

26) H. Scichlein, A. C. Müller, M. Voigts, A. Krügel and E. IversTifée, J. Appl. Electrochem., 32, 875-882 (2002).

27) Wavemetrics, IgorPro (Commercial software), http://www. wavemetrics.com.

28) Scilab Enterprises, Scilab (free and open source software), http://www.scilab.org/.

29) The Python Software Foundation, Python (Open source license software), http://www.python.org.

30) The R Foundation, R (free software environment for statistical computing and graphics), http://www.r-project.org/.

31) J. W. Eaton, GNU Octave (GNU general public license software), http://www.gnu.org/software/octave/.

32) K. Kobayashi and Y. Sakka, Solid State Ionics, 232, 49-57 (2013).

33) P. T. Boggs, H. B. Richard, J. E. Rogers and R. B. Schnabel, User's reference guide for ODRPACK version 2.01 software for weighted orthogonal distance regression, NISTIR 4834. http:// docs.scipy.org/doc/external/odrpack_guide.pdf.

34) J. W. Zwolak, P. T. Boggs and L. T. Watson, Acm Trans. Math. Soft, 33, 27 (2007).

35) S. Bhattacharjee and M. A. Alim, J. Mater. Sci.: Mater. Electron., 26, 4521-4540 (2015).

36) J. R. Taylor, "An introduction to error analysis", 2nd ed, University Science Books, Sausalito (1997).

37) G. A. F. Seber and C. J. Wikd, "Nonlinear regression", John Wiely \& Sons, Inc., New Jersey (2003).

38) S. Mihara, K. Kobayashi, T. Akashi and Y. Sakka, Key Eng. Mater., 616, 120-128 (2014). 